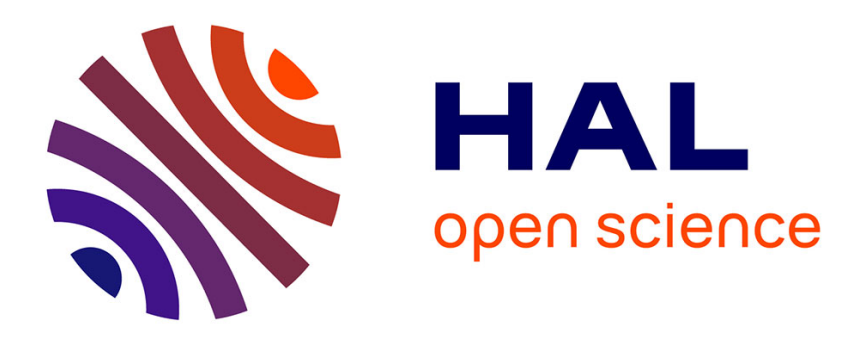

\title{
WAVE PROCESSES IN THERMOVISCOELASTIC MEDIUM
}

\author{
L. Merzhievsky, Y. Kondratyev
}

\section{To cite this version:}

L. Merzhievsky, Y. Kondratyev. WAVE PROCESSES IN THERMOVISCOELASTIC MEDIUM. Journal de Physique IV Proceedings, 1991, 01 (C3), pp.C3-503-C3-510. 10.1051/jp4:1991371 . jpa00250515

\section{HAL Id: jpa-00250515 https://hal.science/jpa-00250515}

Submitted on 1 Jan 1991

HAL is a multi-disciplinary open access archive for the deposit and dissemination of scientific research documents, whether they are published or not. The documents may come from teaching and research institutions in France or abroad, or from public or private research centers.
L'archive ouverte pluridisciplinaire HAL, est destinée au dépôt et à la diffusion de documents scientifiques de niveau recherche, publiés ou non, émanant des établissements d'enseignement et de recherche français ou étrangers, des laboratoires publics ou privés. 


\title{
WAVE PROCESSES IN THERMOVISCOELASTIC MEDIUM
}

\author{
L.A. MERZHIEVSKY and Y.F. KONDRATYEV
}

Lavrentyev Institute of Hydrodynamics, Sibirian Division of the USSR Academy of Sciences, Novosibirsk 630090, USSR

\begin{abstract}
résumé : Une modèle de corps thermoviscoélastique est formulé, incluant l'équation non linéaire hyperbolique de la conduction de la chaleur. I'influence du flux de chaleur et de la température sur le temps de relaxation du flux thermique est prise en compte. Des exemples de solutions de problèmes particuliers sont donnés. les résultats des calculs sont comparés aux résultats expérimentaux.
\end{abstract}

Abstract. A thermoviscoelastic body model is formulated, that comprises the nonlinear hyperbolic heat conductivity equation. A dependence of the thermal flux relaxation time on the magnitude of heat flux and temperature has been built. The examples of solving particular problems are given. The calculation results are compared to the experimenlal ones.

\section{I - INTRODUCTION}

The classical equation of heat conductivity is the equation of parabolic type. Phisically, it corresponds to a hypothesis of the instantaneous heat propagation. It is expedient to be applied for analyzing the processes which velocity is essentially less than that one of heat transfer. For the case of nonstationary high-rate processes, for example, high-rate impact or laser radiation effect on materials, the allowance for the fact that the heat propagation velocity is finite is required. For this reason, to rearrange the temperature field at an abrupt change of a heat flux, we need the finite time called the thermal relaxation time.

The mentioned peculiarities of the processes can be taken into account with the help of the hyperbolic heat conductivity equation. To derive it, the phenomenological Fourier law for the heat flux density is modified. This has been discussed in literature for a long time. Thus, little attention is undeservedly paid to the question of construction of a dependence for the thermal relaxation time. At the best, within 
the framework of either assumption, an order of the value of the thermal relaxation time is estimated. Depending on hypotheses adopted, it can change by orders. Actually, the thermal relaxation time is a function of thermodynamic parameters (for example, entropy and heat flux) and parameters characterising the stress-strain state of the medium. The availability of such function is a necessary condition for construction of a closed model for describing adequately behaviour of materials under extreme conditions. Construction of a simple variant of such a dependence is one of main aims of this paper.

For a model used, the hyperbolic heat conductivity equation is combined with the equations of the viscoelastic Maxwell-like medium, that were applied to solve the problems of dynamic and shock deforma- tion of metals.

\section{II - BASIC RELATIONS OF THE MODEL}

A conventional form of the phenomenological Fourier law connects the heat flux density $q$ and the temperature gradient $T$. For the immovable undeformable medium

$$
\mathrm{q}=-\lambda \operatorname{grad} \mathbf{T} \text {, }
$$

where $\lambda$ is the heat conductivity coefficients. For taking into account the limitedness of the heat propagation velocity, use is made of its modification

$$
q=-\lambda \operatorname{qrad} T-\tau_{1} \frac{\partial q}{\partial t}
$$

where $\tau_{1}$ is the time of thermal flux relaxation. In the general case, the value of $q$ is the vector and $\lambda$ is the second-range tensor. Confining ourselves to consideration of the one-dimensional processes, we'll rewrite (1) for this case as

$$
q=-\lambda \frac{\partial T}{\partial x}-\tau_{1} \frac{\partial q}{\partial t}
$$

To derive the hyperbolic heat conductivity equation, expression (2) is often substituted into the heat balance equation

$$
\rho \frac{\partial E}{\partial x}+\frac{\partial q}{\partial t}=0 \text {. }
$$

giving in this case the second-order equation for T ( $\rho$ is the substance density, $\mathrm{E}$ is the specific internal energy). Such an approach has a series of disadvantages. In particular, owing to the process nonequilibrium, the difficulties arise in introducing the notion of temperature, in analyzing the thermodynamic and mathematical correctness of the system obtained, and in defining the generalized solution of the shock wave type, since equation (2) is not divergent. For the reasons mentioned, to describe the process of heat transfer, let us, as in $[1,2]$, make use of the entropy balance equation and the conservation law for the heat pulse density pq. In the one-demensional nonstationary case, the complete system of equations of thermovisco- elastic medium, that was obtained in [2], has the form 


$$
\begin{aligned}
& \frac{\partial \rho}{\partial t}+\frac{\partial \rho u}{\partial x}=0 \text {; } \\
& \frac{\partial \rho u}{\partial t}+\frac{\partial\left(\rho u^{2}-\sigma_{1}\right)}{\partial x}=0 \text {; } \\
& \frac{\partial \rho h_{2}}{\partial t}+\frac{\partial \rho u h_{2}}{\partial x}=-\rho \psi_{2} \text {; } \\
& \frac{\partial \rho h_{3}}{\partial t}+\frac{\partial \rho u h_{3}}{\partial x}=-\rho \psi_{3} \text {; } \\
& \frac{\partial p q}{\partial t}+\frac{\partial\left(p u q+E_{s}\right)}{\partial x}=-\frac{\rho q}{\tau_{1}} \text {; } \\
& \frac{\partial \rho e}{\partial t}+\frac{\partial\left(\rho u e-u \sigma_{1}+\alpha q E_{s}\right)}{\partial x}=0 \text {; }
\end{aligned}
$$

where $e=E+\frac{u^{2}}{2}+\frac{\alpha q^{2}}{2} ; \quad \psi_{i}=\frac{h_{i}-\frac{\left(h_{1}+h_{2}+h_{3}\right\rangle}{3}}{\tau_{2}}, i=2,3 ;$

$h_{1}, h_{2}, h_{3}$ - are the components of the Henkey deformation tensor in the main axes; $E=E\left(h_{1}, h_{2}, h_{3}, S\right) ; s$ is the entropy; $E_{s}=\frac{\partial E}{\partial S}=T ; \alpha$ is the coefficient connected with the heat transfer velocity; $\tau_{2}=\tau_{2}\left(h_{1}, h_{2}, h_{3}, s\right)$ denotes the relaxation time of shear stresses.

To close system (3), a dependence of the thermal relaxation time $\tau_{1}$ on parameters characterising the medium state is required. Unlike gases, for solids several. heat conductivity mechanisms acting simultaneously can be stated. For estimation of the value of $\tau_{1}$, that is often based on relation

$$
w=\sqrt{\frac{\lambda}{\rho c_{v} \tau_{1}}}=\sqrt{\frac{a}{\tau_{1}}},
$$

( $W$ is the heat propagation velocity, $c_{v}$ is the specific thermal capacity at constant volume, $a$ is the temperature conductivity coefficient), one of probable mechanisms is usually considered to be predominant. As a result, the obtained estimates of the value of $\tau_{1}$ can differ by some orders. Thus, for metals $\tau_{1} \sim 10^{-11} \mathrm{~s}$, as in [3]. In [2] the value of $\tau_{1}$ is assumed to coincide in the order with the electron inertia time $\left(10^{-13} \div 10^{-14} \mathrm{~s}\right)$, and for copper it is taken to 
be equal to $610^{-14} \mathrm{~s}$. This value is close to the period of thermal ion fluctuation (for copper $2.510^{-13} \mathrm{~s}$ ). The estimate from [4], that was obtained with the help of the quantum-mechanical form of the Boltzmann equation for the statistical functions of propagation of free electrons and phonons, gives the value of $\tau_{1} \sim 10^{-14}$. The papers, where the value of $\tau_{1}$ is considered as the function of parameters characterising the medium state, are unknown to the authors.

Let us consider that the change of the value of $\tau_{1}$ is due, in the first place, to the change of thermodynamic parameters. The values of $q$ and $s$ are chosen as the determining parameters, i.e. it is taken that $\tau_{1}=\tau_{1}(q, s)$. With the known $E=E\left(h_{1}, h_{2}, h_{3}, s\right)$ and relation $E_{s}=T$, we can change to a dependence in the form $\tau_{1}=\tau_{1}(q, T)$ that is more convenient for analyzing the qualitative peculiarities of the dependence under investigaton.

With the help of relation $\tau_{1} \lambda, a$, that follows from (4), consider the character of the change of $\tau_{1}$ with temperature. The available information concerning the change of $\lambda$ with $T$ is lack and often contradictory. On the one hand, this is due to difficulties arising at theoretical and experimental determination of $\lambda$, and on the other hand, this is connected with a variety of heat conductivity mechanisms and their intricate combinations.

Depending on the value of $q$, the bodies under consideration can be both in solid, liquid or vapour state. Let us confine ourselves to consideration of solid and liquid phases of metals, that are the most complicated for the analysis. It is known [5], that heat conductivity of different metals (probably, owing to peculiarities of combination of heat conductivity mechanisms) can both decrease and increase with T. In the liquid state, their heat conductivity usually decreases. It is possible to take into account the mentioned peculiarities for the corresponding behaviour of $\tau_{1}$ if we take that

$$
\tau_{1}=\frac{q}{q_{\star}} \exp \left[-\left(\frac{T-T_{0}}{T_{1}}\right)^{n}\right]=\tau_{0} \frac{q}{q_{*}} \exp \left[-\left(\frac{T-T_{0}}{T_{1}}\right)^{n}\right]
$$

where $\tau_{0}, q_{*}, n$ are the constants to be determined by the experimental data; $\mathbb{T}_{0}, \mathbb{T}_{1}$ are the initial temperature and the one of melting, respectively. This choice of the functional form $\tau_{1}=\tau_{1}(q, T)$ is not thought at all as being unambiguous, however, as is seen from further investigations, it allows a qualitative and quantitative description of some phenomena observed experimentally to be made within the framework of a model chosen. In the first place, these are phenomena due to propagation of stress waves induced in metals under the action of not very powerful laser pulse. The experimental data concerning decay of the compression wave induced by laser pulse [6] were the 
basis to choose parameters in (5). For numerical calculations, the same dependences for $E$ and $\tau_{2}$, as in [7], were used

\section{III - RESULTS OF NUMERICAL CALCULATION}

A series of papers is known, that are devoted to calculation of mechanical stresses in metals under the action of laser pulse with the help of different models of material behaviour and those ones of absorption of laser radiation energy in a substance $[8,9]$. Thus, in [9], a temperature dependence of thermal capacity, heat conductivity coefficients and linear expansion is taken into account as well as a dependence of the coefficient of laser radiation absorption and reflecting ability of the specimen surface on temperature and wave length. In this case, confine ourselves to a more simple statement of the problem, that corresponds to experimental conditions from [6]. The density of radiation power is assumed to be such that intense evaporation or melting of metal doesn't occur under its action. The laser pulse effect is simulated by a heat flux at the specimen boundary. The problem is reduced to solution of system (3), (5) with the boundary condition:

$$
\text { for } x=0 \quad q=q(t) ; \quad q(t)= \begin{cases}q_{0} \sin \left(\pi t / t_{1}\right), & t \leqslant t_{1} \\ 0, & t>t_{1}\end{cases}
$$

The experimental parameters from [6] are the following: $t_{1}=50 \mathrm{~ns}, q_{0}$ is the pulse amplitude. The ferrous specimens were under the action of laser pulse. A modification of the Godunov's method was used for numerical solution.

The main parameter measured in the exsperiments [6] is the amplitude of the compression wave generated by laser pulse at different distances from the surface of radiation. In accordance with accuracy of description of the experimental data on the mentioned parameter, the choice of constants in (5) was made. In Fig. 1 the typical spatial distribution of the stress $\sigma_{1}$, obtained in calculations, is illustrated for different time (for curves $1-3, t=0.2 ; 0.4 ; 0.5$; respective$1 y)$. This distribution is characterized by qualitative peculiarities that can be found in calculations of other researchers [9]: initially, a compression pulse goes into the undisturbed material, after which the tensile stress pulse propagates beginning from the moment when the laser pulse action stops. The both pulses decay little by little with propagation. The chosen values of parameters in (5) provide rather accurate calculational representation of the experimental compression pulse decay curve, obtained in [6]. To resolve an ambiguity in determination of the dependence parameters [5], use was made of the heat propagation velocity estimate obtained from (5) and (4). The chosen values of the parameters $n=2 \div 3, q_{*}=10 ., \tau_{0}=310^{-8}$ s provide a plausible values of $W$. 
The results discussed are presented in Fig. 2 , where the points show the experimental data, 1 - the calculation with (5) at the values of parameters given. During calculation, the time of relaxation of the heat flux ranges from $10^{-13} \leqslant \tau_{1} \leqslant 10^{-8} \mathrm{~s}$. In this connection the available estimates $\tau_{1} \sim 10^{-11}$ are thought to be close to a certain average value of $\tau_{1}(q, T)$, and $\tau_{1}=10^{-13} \div 10^{-14} \mathrm{~s}$ - to minimum one. It is important that description of the experimental compression pulse decay curve at any constant value of $\tau_{1}$ was not a success. Figure 2 shows the calculation results for the constant value of $\tau_{1}$ : curve $2-3$ $10^{-8} \mathrm{~s} ; 610^{-9} \mathrm{~s}$, curve $3-310^{-9} \mathrm{~s}$. As is seen from calculation results presented in Figure, the curves with $\tau_{1}=$ const $=c_{1}$, where $10^{-8} \leqslant c_{1} \leqslant 6$ $10^{-9}$, almost coincide. The further decrease in $\tau_{1}$ leads to displacement of the curve, describing the decay, upwards with respect to curve 2 (for example, curve 3). For comparison, Fig. 2 presents the result of calculation [6] by the model, that takes into account material evaporation and is based on the corresponding equation of state. From comparison it is seen that this calculation doesn't give not only quantitative and also qualitative description of the decay process.

For further verification of the model, the problem of uniform deformation of a thin ferrous rod under the action of a heat flux is solved as well as the one of rod extension with constant strain rate and under simulataneous action of the heat flux. The statement of such zerodimensional problem was discussed in [10]. In this case the functions entering (4), (5) depend only on $t$, and the system of equations itself is reduced to the system of ordinary differential equations of rigid type, for numerical solution of which special methods [10] are required. The results of solution of the first case are shown in Fig 3. ( $a$ is the temperature change with time, $b$ is the change of the stress $\sigma_{1}$ with time). The heat flux value was chosen such that the rod heated up to $\mathrm{T}=370^{\circ} \mathrm{K}, 1060^{\circ} \mathrm{K}, 1500^{\circ} \mathrm{K}$ (curves $1-3$, respectively). In this case, as Figure shows, the time of attaining the steady temperature value changed. On the nonequilibrium part, an abrupt overshoot of the longitudinal compression stress was observed, since the nondisplacement of the rod in this direction does't allow the thermal expansion of the specimen. Further on, the stresses relax to the stationary value characteristic of this temperature.

In the second case, when the rod being heated deforms with a constant strain rate, that is equal to $10^{3} \mathrm{~s}^{-1}$, at different heat fluxes (and, respectively, at the same eguilibrium temperatures as in Fig. 3), behaviour of the dependences $\sigma_{1}(t)$ (that is equivalent to the dependence $\sigma_{1}\left(\varepsilon_{1}\right)$, were $\varepsilon_{1}$ is the deformation) is different. At minimum heat flux the dependence $\sigma_{1}(t)$ has the form characteristic of an ideal elastoplastic body (Fig. 4 , curve 1). At heating up to $1060^{\circ} \mathrm{K}$, the stress initially increases and then relaxes to the stationary value (Fig. 4, curve 2). Of interest is the behaviour of $\sigma_{1}(t)$ at heating up to $1500^{\circ} \mathrm{K}$ (Fig. 4 , curve 3 ): initially the stress increases, then drops abruptly and becomes compressing, after this it increases again 

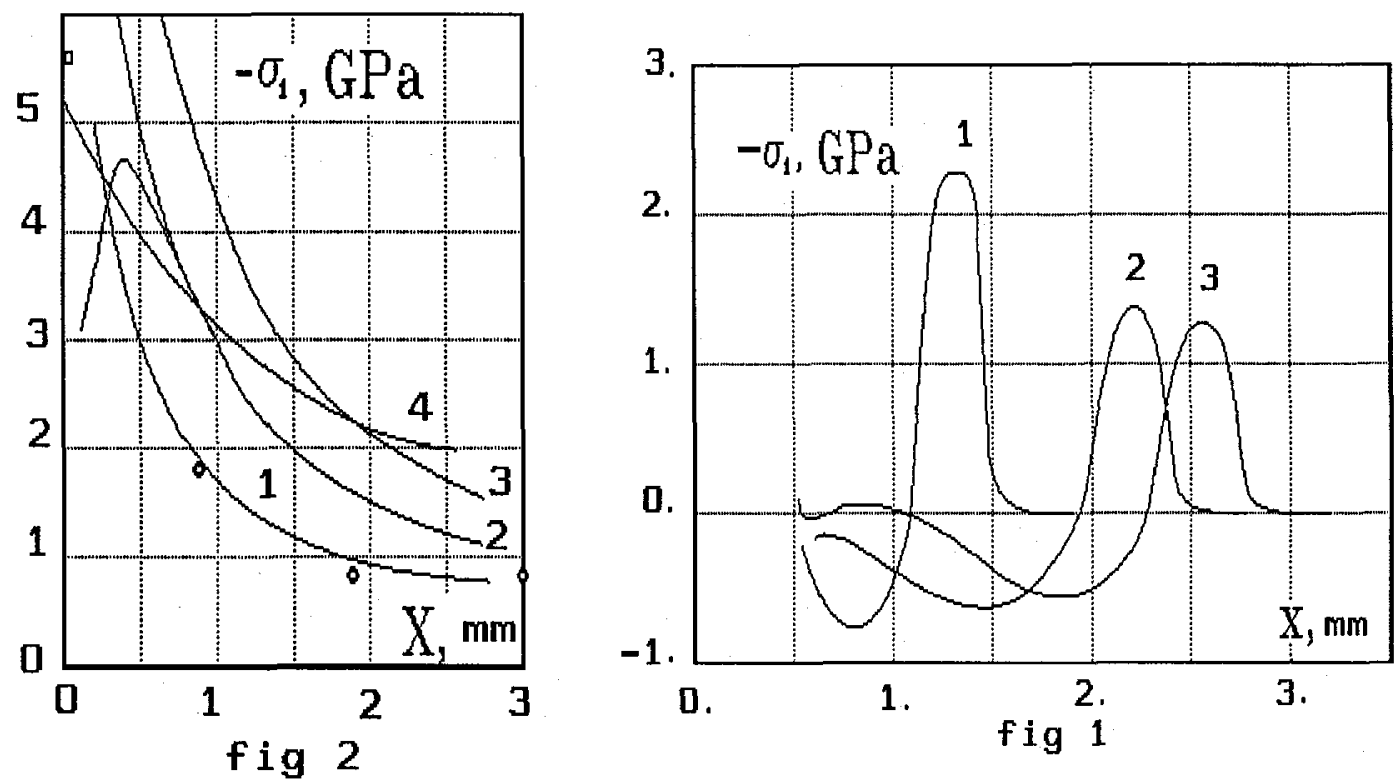

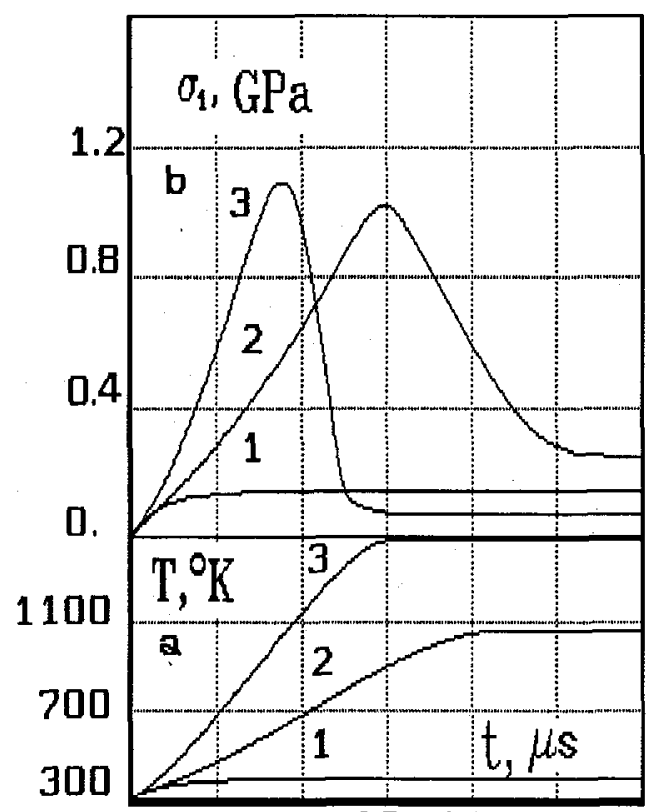

0. 4. B. 12, 16, 20 . fig 3

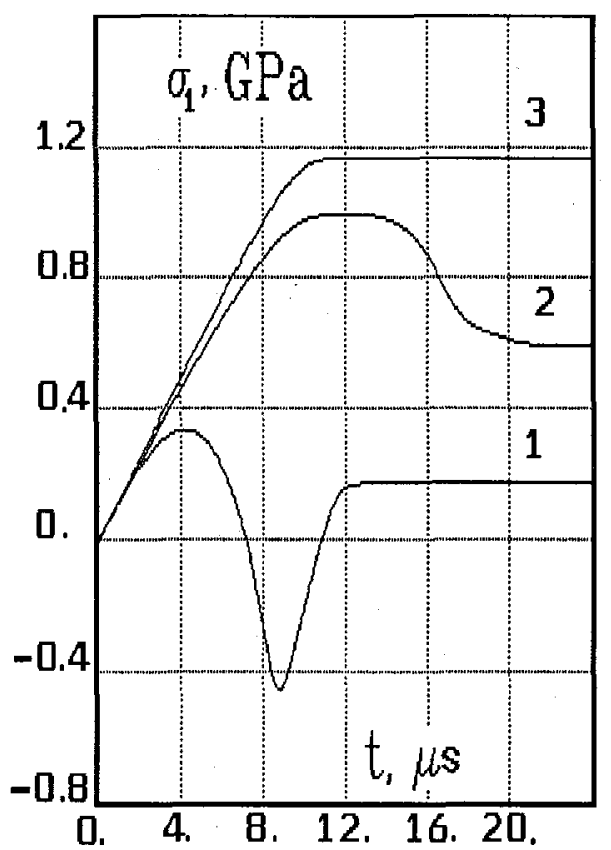

fig 4 
to the stationary value. Such a form of the dependence $\sigma_{1}(t)$ is due to that the strain rate value of $10^{3} \mathrm{~s}^{-1}$ turns out to be less than the thermal expansion velocity. As a result, the thermal expansion is retarded by the slowed-down body motion. In all the cases the stationary value of $\sigma_{1}$ is equal to the value of yield point, that corresponds to the given strain rate and temperature [10]. This result assures a validity of description of the strength properties of materials under high srtain rates and temperatures.

\section{IV - CONCLUSION}

The paper presents a formulation of a thermoviscoelastic body model comprising the nonlinear hyperbolic heat conductivity equation. A dependence of the thermal flux relaxation time on the heat flux and temperature has been built. This allows rather peculiar nonstationary processes, that are due to the nonequilibrium of the heat fluxes at large temperature gradients, to be described. The numerical calculation results show that the model can be applied to calculate the wave processes induced by the intense heat action.

\section{REFERENCES}

1. Malyshev A.N., Romensky E.I. Sib. Math. Journ. $27(1986) 5,128$.

2. Romensky E.D. Sib. Math. Journ. 30(1989) 4,135

3. Lykov A.V. Heat conductivity theory. Moscow, Vysshaya shkola, (1967),600.

4. Michel J.M. JAP, 40 (1969) 13, 5123 .

5. Bosworth R.C.I. Heat transfer phenomena. Moscow, (1957), 275.

6. Fairand B.P. et ol. Appl. Phys. letters, 25(1974)8,431.

7. Merzhievsky L.A., Resnyansky A.D. Journ. de Physique, $46(1985) 8,67$.

8. Mirkin L.I. Physical foundations of laser beam treatment of materials. Moscow, MGU, (1975), 383 .

9. Avrasin E.T., Kesselman V.S., Frolov A.B. Calculation of mechanical stresses in metals under the action of laser pulse. Fizika i Khimiya Obrabotki Materialov, (1985)1,43.

10. Merzhievsky L.A., Shamonin S.A. PMTF. (1980) 5,170. 